\title{
Modeling and Numerical Analysis of the Solid Particle Erosion in Curved Ducts
}

\author{
Ke Sun, ${ }^{1,2}$ Lin Lu, ${ }^{1}$ and Hanhui Jin ${ }^{2}$ \\ ${ }^{1}$ Faculty of Construction and Environment, The Hong Kong Polytechnic University, Hung Hom, \\ Kowloon, Hong Kong \\ ${ }^{2}$ School of Aeronautics and Astronautics, Zhejiang University, Hangzhou 310027, China \\ Correspondence should be addressed to Lin Lu; vivien.lu@polyu.edu.hk and Hanhui Jin; enejhh@emb.zju.edu.cn
}

Received 9 September 2013; Accepted 18 October 2013

Academic Editor: Jianzhong Lin

Copyright (C) 2013 Ke Sun et al. This is an open access article distributed under the Creative Commons Attribution License, which permits unrestricted use, distribution, and reproduction in any medium, provided the original work is properly cited.

\begin{abstract}
This paper presents a modeling and computational study on particle erosion in curved ducts. It is found that the average erosion rates per impact range from $4.2 \times 10^{-7}$ to $9.5 \times 10^{-3} \mathrm{~mm}^{3} / \mathrm{g}$ under current conditions. For each doubled inlet velocity, the increases of erosion rates per impact are 2-14 times. The erosion rate per impact varies with particle diameter with " $\sqrt{ }$ " shape through bends, which is similar to the particle deposition behavior in duct flows. The erosion rate curves per injected particle show the shapes of a 90-degree anticlockwise rotated "S" and a wide open "V," respectively, for three larger and smaller inlet velocities. The average erosion rates per injected particle are 1.4-18.9 times those rates per impact due to huge amounts of impacting, especially for those depositing particles. It is obvious that the erosion rate distribution per impact is similar to a "fingerprint" with five clear stripes and a lower "cloud" along the bend deflection angle for the three largest particles; yet, for other smaller particles, the erosion rate distributions are much like an entire "cloud."
\end{abstract}

\section{Introduction}

Particulate flow is a significant phenomenon in environmental, industrial, medical, and lifetime applications. For example, the conveying and ventilation system is severely affected by particle flow and its erosion [1-3]. The aerosol deposition, accumulation, and soiling on solar panels and glazing glass will erode their surfaces obviously [4]. These applications also include a large amount of straight and curved duct flow. However, the particle flow and erosion in bends have not been fully studied $[5,6]$.

El-Behery et al. [7] studied the penetration rate distribution for estimating solid particle erosion in curved $90^{\circ}$ and $180^{\circ}$ ducts. Sun et al. [8] studied the particle penetration and deposition in and behind bends. Chen et al. [9] proposed a comprehensive procedure to estimate the erosion in elbows mainly for bubbly or droplet flow. Zhang et al. [10] adopted Computational Fluid Dynamics (CFD) with near-wall and volume improvement to predict particle impact in a sharp bend. Macchini et al. [11] investigated the influence of particle size, density, and concentration on bend erosive wear under high particle concentrations. Although some studies have been conducted to investigate the particle erosion in bends, prediction research on the erosion distribution is limited.

Therefore, present study focuses on the erosion status modeling and analysis of curved walls. The airflow and particle flow are modeled and predicted to obtain the erosion information in typical 90-degree bends. The behaviors of erosion rates varying with particle diameter or Stokes number, inlet velocity, and deflection angles are analyzed in detail.

\section{Method}

2.1. Fluid and Particle Flow Model. Before solving the particle flow in a fluid, the fluid flow conservation equations were deducted and predicted by CFD tools [12]. Together with near-wall two-layer model, the Reynolds stress model (RSM) 
was utilized to solve the fluid flow system in the curved duct due to its ability to predict the swirling, turbulent, and near-surface flow [8]. Details of the RSM model are given as follows:

$$
\begin{gathered}
\frac{\partial \bar{u}_{i}}{\partial x_{i}}=0 \\
\frac{\partial \bar{u}_{i}}{\partial t}+\bar{u}_{j} \frac{\partial \bar{u}_{i}}{\partial x_{j}}=-\frac{1}{\rho} \frac{\partial \bar{p}}{\partial x_{i}}+\frac{1}{\rho} \frac{\partial}{\partial x_{j}}\left(\mu \frac{\partial \bar{u}_{i}}{\partial x_{j}}-\rho \overline{u_{i}^{\prime} u_{j}^{\prime}}\right),
\end{gathered}
$$

where $\bar{u}_{i}$ is the mean velocity, $r$ and $m$ are the density and molecular viscosity of the fluid, respectively, $\bar{p}$ is the mean pressure, and $-\rho \overline{u_{i}^{\prime} u_{j}^{\prime}}$ is the Reynolds stress. The general transport equations for the Reynolds stresses could be estimated by

$$
\begin{aligned}
& \frac{\partial}{\partial t}\left(\rho \overline{u_{i}^{\prime} u_{j}^{\prime}}\right)+\frac{\partial}{\partial x_{k}}\left(\rho \overline{u_{k}} \overline{u_{i}^{\prime} u_{j}^{\prime}}\right) \\
&=-\frac{\partial}{\partial x_{k}}\left[\rho \overline{u_{i}^{\prime} u_{j}^{\prime} u_{k}^{\prime}}+\overline{p\left(\delta_{k j} u_{i}^{\prime}+\delta_{i k} u_{j}^{\prime}\right)}\right] \\
&+\frac{\partial}{\partial x_{k}}\left[\mu \frac{\partial}{\partial x_{k}}\left(\overline{u_{i}^{\prime} u_{j}^{\prime}}\right)\right]-\rho\left(\overline{u_{i}^{\prime} u_{k}^{\prime}} \frac{\partial \overline{u_{j}}}{\partial x_{k}}+\overline{u_{j}^{\prime} u_{k}^{\prime}} \frac{\partial \overline{u_{i}}}{\partial x_{k}}\right) \\
& \\
&-\rho \beta\left(g_{i} \overline{u_{j}^{\prime} \theta}+g_{j} \overline{u_{i}^{\prime} \theta}\right)+p\left(\frac{\partial u_{i}^{\prime}}{\partial x_{j}}+\frac{\partial u_{j}^{\prime}}{\partial x_{i}}\right) \\
&-2 \mu \frac{\partial u_{i}^{\prime}}{\partial x_{k}} \overline{\partial x_{k}} \\
&-2 \rho \Omega_{k}\left(\overline{u_{j}^{\prime} u_{m}^{\prime}} E_{i k m}+\overline{u_{i}^{\prime} u_{m}^{\prime}} E_{j k m}\right)+S_{\mathrm{user}},
\end{aligned}
$$

where the 1st and 4th-6th terms on the right of this equation have detailed models to close this equation as that in [12]. Neumann outlet flow conditions were employed and the walls were set as smooth. Staggered numerical scheme was adopted for pressure equation and second-order discretization methods were applied for other variables. These variable convergences were achieved when their residuals were $10^{-5}$ or less.

Based on the modeling and computation of fluid flow field, the particle flow was determined by tracking the path of each particle with the Lagrangian method. When using this method, particles were assumed to be spherical solid ones with diluted flow. The Lagrangian equation can be expressed as

$$
\frac{d u_{p i}}{d t}=F_{D}\left(u_{i}-u_{p i}\right)+g_{i}\left(1-\frac{\rho_{a}}{\rho_{p}}\right)+F_{a i},
$$

where $u_{p i}$ is particle velocity in the $i$ th direction $(\mathrm{m} / \mathrm{s})$ and $u_{i}$ represents the air velocity. The 1 st term on the formula right represents the particle drag force in the $i$ th direction $\left(\mathrm{m} / \mathrm{s}^{2}\right)$, and the 2 nd one is the gravitational force. The 3rd term stands for other possible forces [13]. To simplify the modelling of particle forces, this article only adopts lift force to predict the particle flow through curved duct [14]. The random fluctuation effects of the fluid flow on particles were modeled by "Eddy lifetime" method [15].

2.2. Particle Wall Interaction and Erosion Models. When particles flow along constrained domains, they will interact with different surfaces. For present solid particles, they would deposit on, rebound from, or reimpact with wall surfaces. To model this process, an algebraic particle-wall impact model [16] was utilized to describe the interaction process between particle and wall as follows:

$$
\begin{gathered}
e=-\frac{v_{2, n}^{p}}{v_{1, n}^{p}}=R_{p w}\left(1-\rho_{p w}\right), \\
v_{2, n}^{p}=-e v_{1, n}^{p}, \\
v_{2, t}^{p}=v_{1, t}^{p}-\mu_{1}(1+e) v_{1, n}^{p},
\end{gathered}
$$

where $e$ is the restitution coefficient, $v_{1, n}^{p}$ and $v_{2, n}^{p}$ are particle normal incident and reflected velocities, respectively, $R_{p w}$ stands for the coefficient of restitution without adhesion, $\rho_{p w}$ is the coefficient of adhesion, and $\mu_{1}$ is the ratio of tangential to normal impulse. This model was integrated into the main particle computation by in-house codes.

When particle-wall impact happens, the wall will be eroded by impacted particles gradually. The wall surface erosion rate or wastage rate is commonly defined as the ratio of wall eroded mass or volume to that of impacted particles. The erosion rate is usually determined by particle impacting velocity and angle, wall and particle materials, and their temperatures. In this paper, a convenient model developed by Menguturk et al. $[17,18]$ was introduced into the curved duct flow to analyse the wall erosion condition on the curved duct walls as follows:

$$
\begin{aligned}
E_{v}= & 1.63 \times 10^{-6}\left(v_{1}^{p} \cos \alpha\right)^{2.5} \sin \left(\frac{\pi \alpha}{2 \alpha_{c}}\right) \\
& +4.68 \times 10^{-7}\left(v_{1}^{p} \sin \alpha\right)^{2.5} \quad\left(\alpha \leq \alpha_{c}\right), \\
E_{v}= & 1.63 \times 10^{-6}\left(v_{1}^{p} \cos \alpha\right)^{2.5} \\
& +4.68 \times 10^{-7}\left(v_{1}^{p} \sin \alpha\right)^{2.5} \quad\left(\alpha>\alpha_{c}\right),
\end{aligned}
$$

where the unit of erosion rate $E_{v}$ is $\mathrm{mm}^{3} / \mathrm{g}, v_{1}^{p}$ is the incident impact velocity, $\alpha$ is the incident impact angle near the wall, and $\alpha_{c}$ is the critical incident angle with a constant value of $22.7^{\circ}$. In these formulas, the erosion rate $E_{v}$ has a power relationship with index 2.5 with impact velocity $v_{1}^{p}$. This model was incorporated into the particle-wall interaction model and main particle flow model to obtain the impact and erosion information in the curved duct flow. 


\section{Results and Discussion}

In this paper, the background fluid was selected as air, and the flow domain was formed in the typical curved twodimensional duct with a 90-degree bend as shown in Figure 1. Since particles mainly deposit and impact on the outer bend wall, the erosions are also observed to happen at these places. The deflection angle $\theta$ is also demonstrated from $0^{\circ}$ to $90^{\circ}$ in this figure. Eight particle groups were injected into the duct flow from the duct inlet with even space distribution, which have the diameters $d_{p}=1,3,7,16,30,60,100$, and $200 \mu \mathrm{m}$. At the duct inlet, 60000 particles were injected. The volume fraction is less than $1.02 \times 10^{-6}$. Six inlet velocity $u_{\text {in }}$ conditions were adopted in this analysis, that is, $2.2,5.3,9,15,33$, and $52 \mathrm{~m} / \mathrm{s}$. Totally, more than 48 different conditions were analyzed. The airflow and particle flow models were validated by experimental data through previous works $[8,19]$ in terms of the air and particle velocity profiles, and particle depositions and penetrations, which support the present investigations.

3.1. Erosion Rate per Impact on Curved Walls. Based on the airflow and particle flow modeling and prediction, statistic results of particle-wall impact and erosion status are given in Figure 1. This figure shows the average erosion rate per impacted particle on the curved wall for each inlet velocity and particle diameter conditions. Using the concept of erosion rate per impacted particle can have more physical meanings and higher application abilities.

Generally, the average erosion rates shown in Figure 2 range from $9.5 \times 10^{-3}$ to $4.2 \times 10^{-7} \mathrm{~mm}^{3} / \mathrm{g}$ for the inlet velocity from $52 \mathrm{~m} / \mathrm{s}$ to $2.2 \mathrm{~m} / \mathrm{s}$. Based on these erosion rate results, the wall wastage can be calculated out considering the injected particle mass. The erosion rate changes with inlet velocity sharply as demonstrated in the figure. The inlet velocity conditions are roughly set around 2-time increase from one smaller inlet velocity to another bigger one. However, the erosion rate generally increases nearly 2 to 14 times. This phenomenon can be explained from the erosion rate equation in (5). Although the erosion rate has a power relationship with impact velocity with index 2.5 , the erosion rate has much more complicated nonlinear correlation with inlet velocity due to the complex curved duct flow conditions.

In terms of the changing with particle diameter, the general trend of erosion rate is similar for particle diameter from $d_{p}=1$ to $200 \mu \mathrm{m}$ for different inlet velocities. Firstly, the erosion rate decreases with particle diameter increase. When particle diameter $d_{p}$ is 7 or $16 \mu \mathrm{m}$, the erosion rate gets to the lowest value. After that, it increases with the particle diameter increase. For very large flow inlet velocity and particle diameter, like $d_{p}=200 \mu \mathrm{m}$ at $u_{\text {in }}=33$ and $52 \mathrm{~m} / \mathrm{s}$, the erosion rate decreases a little due to the large inertia and gravity of these large particles with large Stokes number St. These phenomena are similar to that observed in the particle deposition behavior with " $\checkmark$ " shape in duct flows $[20,21]$. This phenomenon may be attributed to the similar impact mechanism during the deposition, rebounding, and reimpaction process. Smaller particles of $d_{p}=1$ to $3 \mu \mathrm{m}$

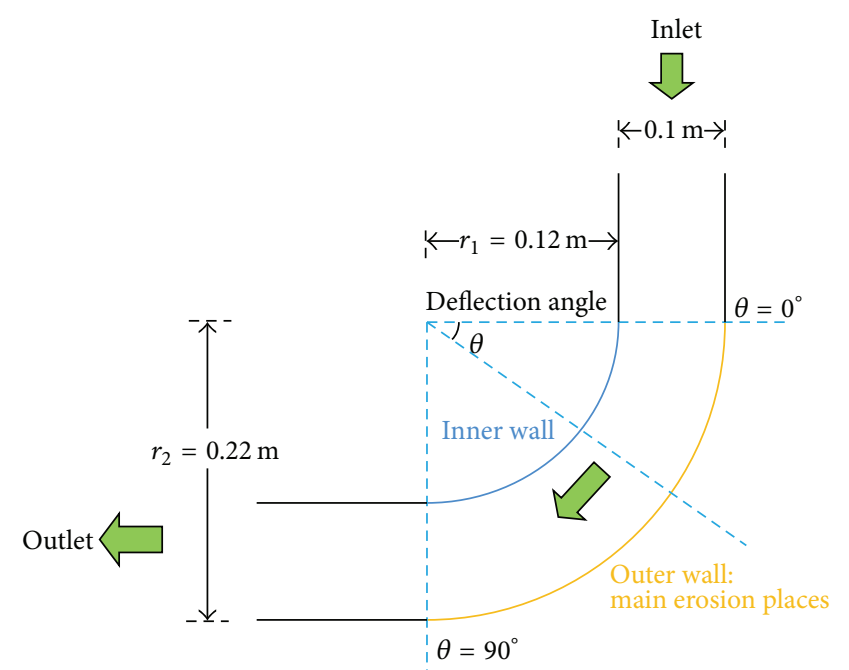

FIGURE 1: Schematic chart of the model geometry.

are affected more by diffusion, turbulence, and vortex in the bend and they may have higher impact angle, and thus higher erosion rates are observed. Coarse ones of $d_{p}=30$ to $200 \mu \mathrm{m}$ are controlled by inertia and gravity, and thus they would erode more wall masses.

3.2. Erosion Rate per Injected Particle on Curved Walls. Since particles may rebound and reimpact onto walls due to the near-wall forces or mechanism like turbulence and vortex, average erosion rate per impacted particle cannot give macroinformation about the injected particle erosion ability. Therefore, Figure 3 shows the average erosion rate per injected particles statistically against the particle Stokes number. The Stokes number St is a key parameter to describe the particle response to fluid flow in bends. This dimensionless number is determined by the following formula:

$$
\mathrm{St}=\frac{\tau_{p}}{\tau_{b}}=\frac{\tau_{p} u_{\mathrm{in}}}{\left(D_{h} / 2\right)}=\frac{C_{c} \rho_{p} d_{p}^{2}}{18 \mu} \cdot \frac{u_{\mathrm{in}}}{\left(D_{h} / 2\right)},
$$

where $\tau_{p}$ is the particle relaxation time, $\tau_{b}$ is the time scale of the bend, $u_{\text {in }}$ is the air velocity at the duct inlet, $D_{h}$ is the hydraulic diameter of the duct, $C_{c}$ is the Cunningham slip correction factor for microparticles, $\rho_{p}$ and $d_{p}$ are particle density and diameter, respectively, and $\mu$ is the air dynamic viscosity. The friction velocity, $u^{*}$, shown in Figure 3 could be calculated by

$$
\begin{aligned}
u^{*} & =u_{\text {in }} \sqrt{\frac{f}{2}}=\frac{u_{\text {in }} / \sqrt{2}}{-3.6 \log \left[6.9 / \operatorname{Re}+\left(k^{\prime} / 3.7 D_{h}\right)^{1.11}\right]} \\
& =-\frac{u_{\text {in }}}{5.1 \log \left[6.9 / \operatorname{Re}+\left(k^{\prime} / 3.7 D_{h}\right)^{1.11}\right]},
\end{aligned}
$$

where $f$ is the Fanning friction factor determined in straight duct by White [22], $k^{\prime}$ is the average microscale roughness height of the rough wall, which is zero in this work for smooth walls, and Re is the Reynolds number. 


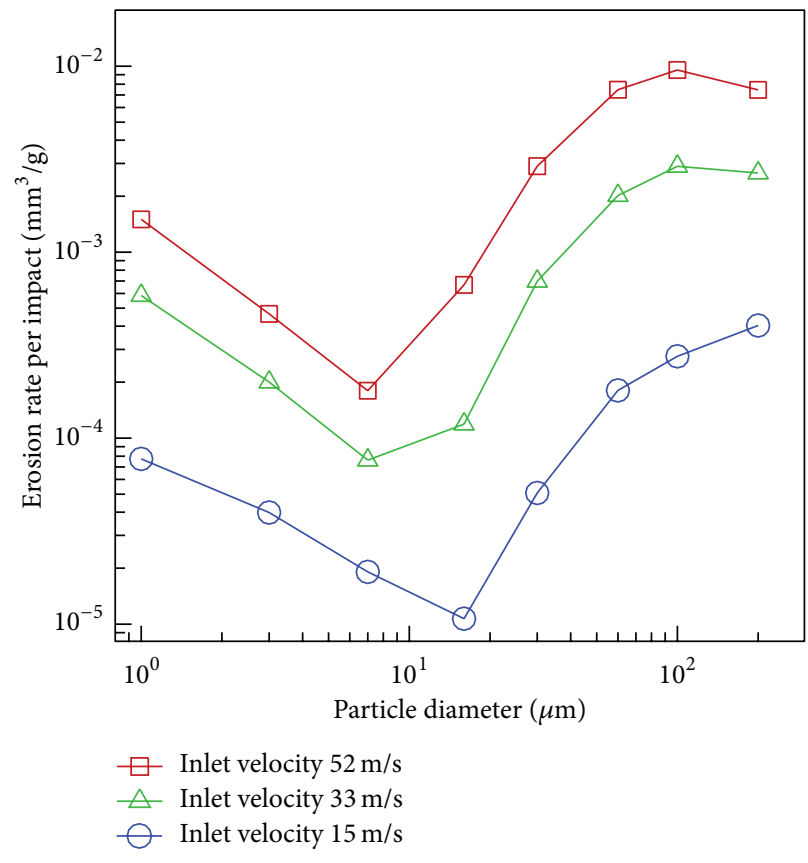

(a)

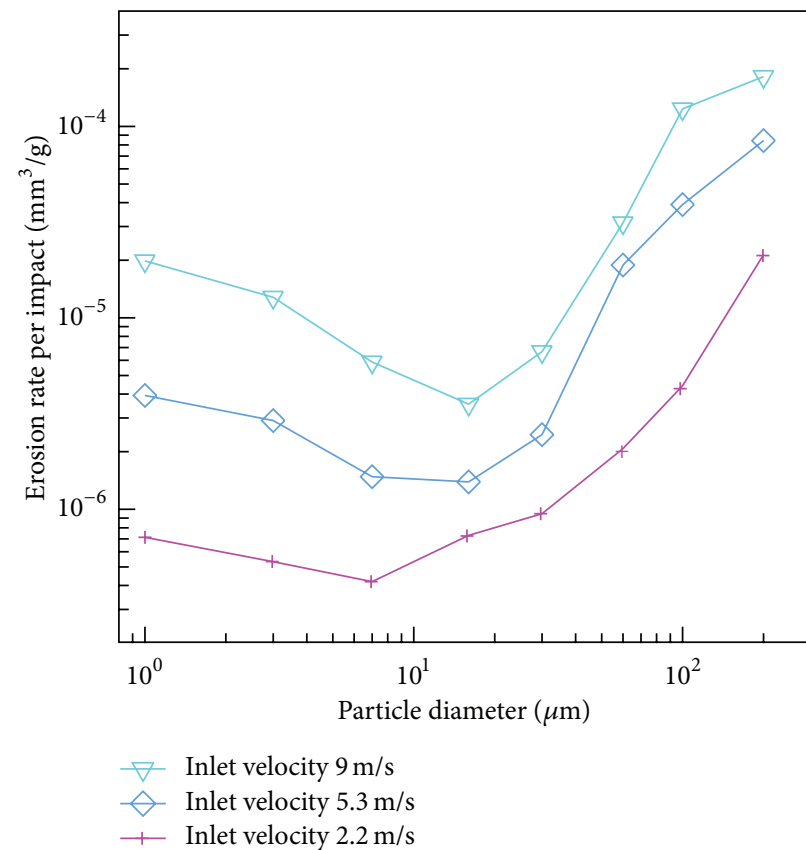

(b)

FIGURE 2: Comparison of the erosion rate $E_{v}$ per impact particle against the particle diameter and inlet flow velocity: (a) larger inlet velocities of 15,33 , and $52 \mathrm{~m} / \mathrm{s}$ and (b) smaller inlet velocities of $2.2,5.3$, and $9 \mathrm{~m} / \mathrm{s}$.

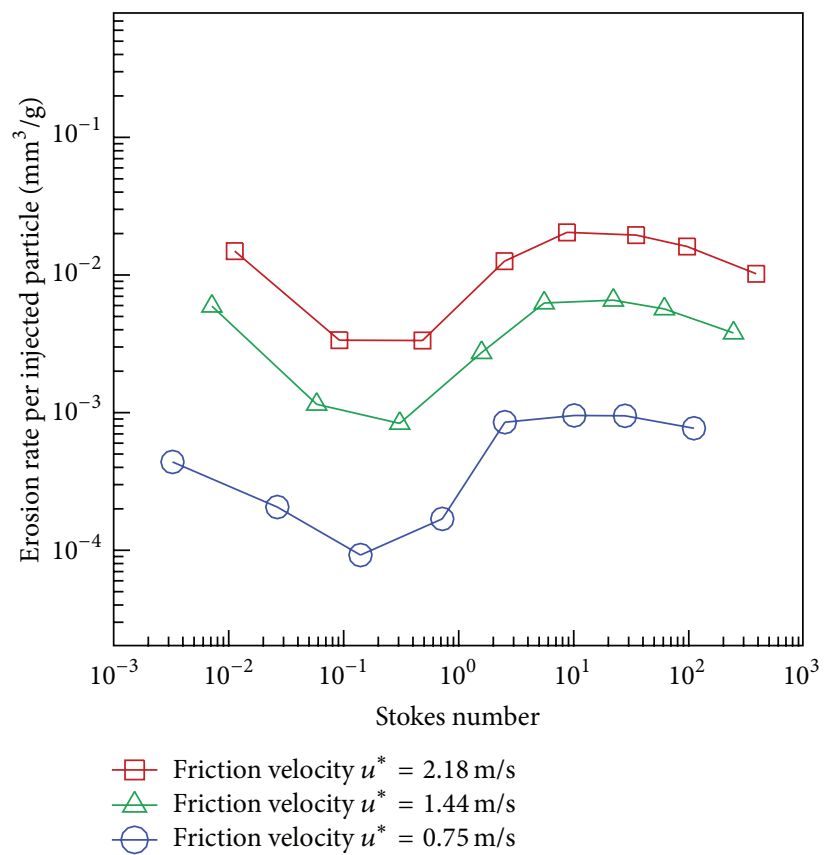

(a)

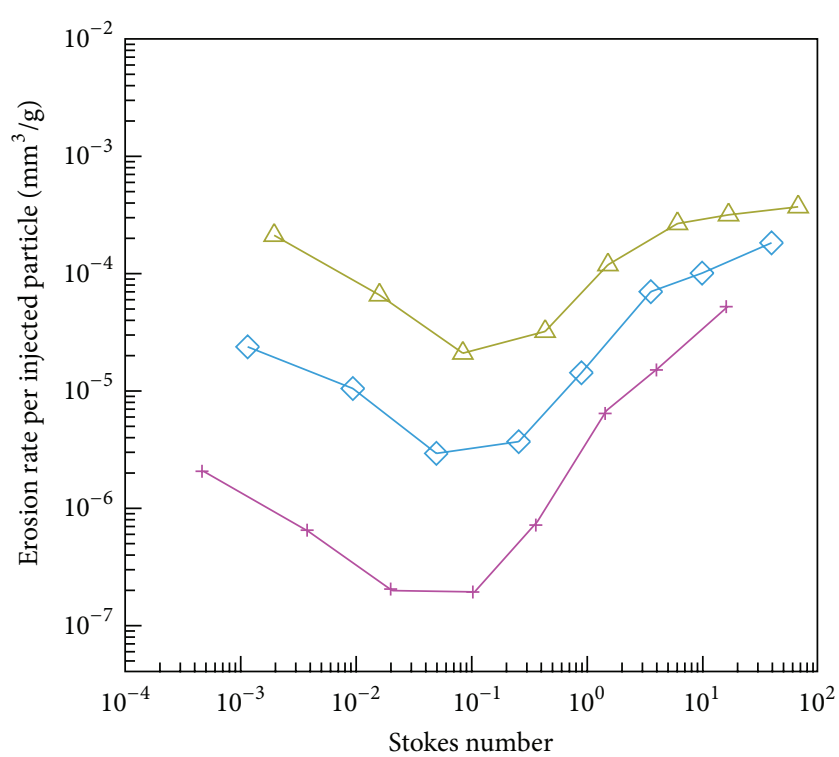

$\triangle$ Friction velocity $u^{*}=0.45 \mathrm{~m} / \mathrm{s}$

$\triangle$ Friction velocity $u^{*}=0.28 \mathrm{~m} / \mathrm{s}$

+ Friction velocity $u^{*}=0.13 \mathrm{~m} / \mathrm{s}$

(b)

FIGURE 3: Comparison of the erosion rate $E_{v}$ per injected particle against the particle diameter and inlet flow velocity: (a) larger inlet velocities of 15,33 , and $52 \mathrm{~m} / \mathrm{s}$ and (b) smaller inlet velocities of $2.2,5.3$, and $9 \mathrm{~m} / \mathrm{s}$. 


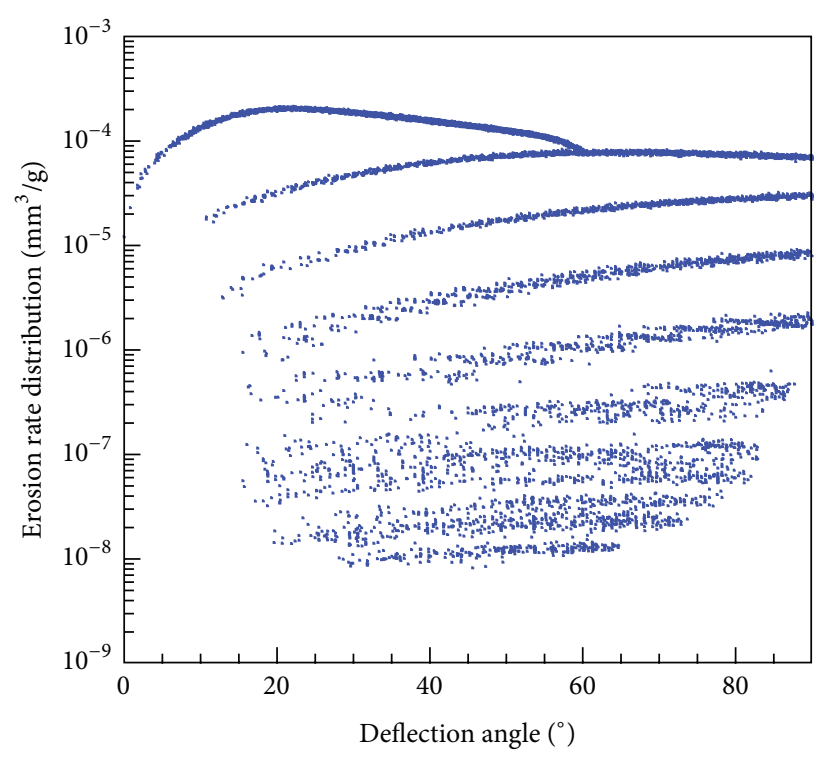

(a)

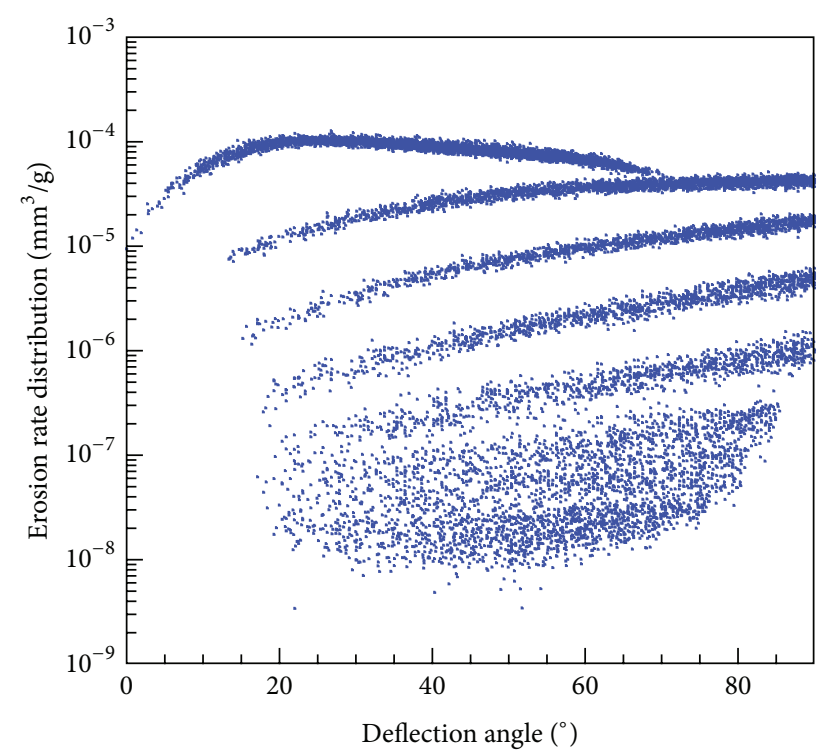

(b)

FIGURE 4: Erosion rate $E_{v}$ distribution per impact particle against the deflection angle of curved duct at the inlet velocity $5.3 \mathrm{~m} / \mathrm{s}$ : (a) particle diameter $200 \mu \mathrm{m}$ and (b) particle diameter $100 \mu \mathrm{m}$.

The erosion rate curves shown in Figure 3 are generally smoother than those in Figure 2. The curve shapes in Figure 3(a) are much like a 90-degree anticlockwise rotated "S." The erosion rates range from $9.2 \times 10^{-5}$ to $2.0 \times$ $10^{-2} \mathrm{~mm}^{3} / \mathrm{g}$. The magnitudes of the values are generally much larger than those in Figure 2(a) due to huge amounts of impacting, especially for those depositing particles. For a similar condition, the erosion rates in Figure 3(a) are 1.4-18.9 times those in Figure 2(a). If the solid particles have high velocities and will deposit onto the bend surfaces, they may impact, rebound, and reimpact on the bend walls to gradually lower down its velocity for deposition. Furthermore, since particles will deposit more on ducts for larger inlet velocities or friction velocities as demonstrated in previous research $[19,20]$, the erosion rates in Figure 3(a) are generally high. In addition, smaller particles of Stokes number $\mathrm{St}=3.3 \times 10^{-3}$ $7.2 \times 10^{-1}$ have higher impaction frequencies or amounts than the other larger ones as shown in the figure. This behavior may be due to their frequent interaction with airflows.

For lower inlet velocity cases shown in Figure 3(b), the erosion rate curves are much like wide open " $\mathrm{V}$ " shapes. If larger particles of $d_{p}>200 \mu \mathrm{m}$ are simulated, the curves may become the shapes demonstrated in Figure 3(a). The erosion rates range from $1.9 \times 10^{-7}$ to $3.7 \times 10^{-4} \mathrm{~mm}^{3} / \mathrm{g}$. Most of these erosion rates are still larger than those shown in Figure 2(b) due to multi-impaction of a single depositing particle. The increasing degrees in this figure are smaller than those in Figure 3(a). For friction velocity $u^{*}=0.13 \mathrm{~m} / \mathrm{s}$, the increase degree is the smallest. Furthermore, three erosion rates are smaller than those in Figure 2(b) for the particle groups of St $=0.02,0.11,0.37$. This phenomenon may be attributed to the low particle impaction amount and low particle deposition for these particles with the smallest friction velocity.
3.3. Erosion Rate Distribution for Each Impact. Distribution of the erosion rate per impacted particle can further demonstrate the particle erosion picture on bend walls. Detailed erosion magnitude and locations can be interpreted. Particle depositions in bends mainly happen on the outer bend walls as shown in Figure 1 and previous research results [23]. Therefore, the following analysis primarily states the erosions on different locations of the outer bend wall.

Figures 4-7 show the distribution of erosion rate $E_{v}$ per impacted particle against the deflection angle as demonstrated in Figure 1. The angles from $0^{\circ}$ to $90^{\circ}$ represent bend inlet to outlet. The case of the inlet velocity $u_{\text {in }}=5.3 \mathrm{~m} / \mathrm{s}$ is selected as an example to explain the erosion rate distribution. Figure 4 presents the erosion rate for the largest particles with diameters $d_{p}=100$ and $200 \mu \mathrm{m}$. It is very obvious that the erosion rate is like a "fingerprint" with clear stripes. These stripes are probably caused by the clear impactions of particles. The top stripe is the first impact of particles on the outer bend wall with the largest erosion rate. Following the airflow, the deflection angles with first impaction are from $0^{\circ}$ to $60^{\circ}$, where the peak erosion rate appears at around $20^{\circ}$. After this impaction, four much clear impactions are observed but with lower erosion rate due to moment or velocity reduction after each impaction. The lowest unclear cloud-like areas are the lightest impactions close to particle depositions. They are more randomly distributed due to turbulence, impaction, and diffusion. Deeper color represents that the erosion rates have a large amount at these areas. It can be seen that the particles in this figure have very high erosion rates from $E_{v}=1.0 \times 10^{-5}$ to $1.0 \times 10^{-4} \mathrm{~mm}^{3} / \mathrm{g}$. Furthermore, the "fingerprint" phenomenon in this figure indicates that further impactions would happen after the bend because the 


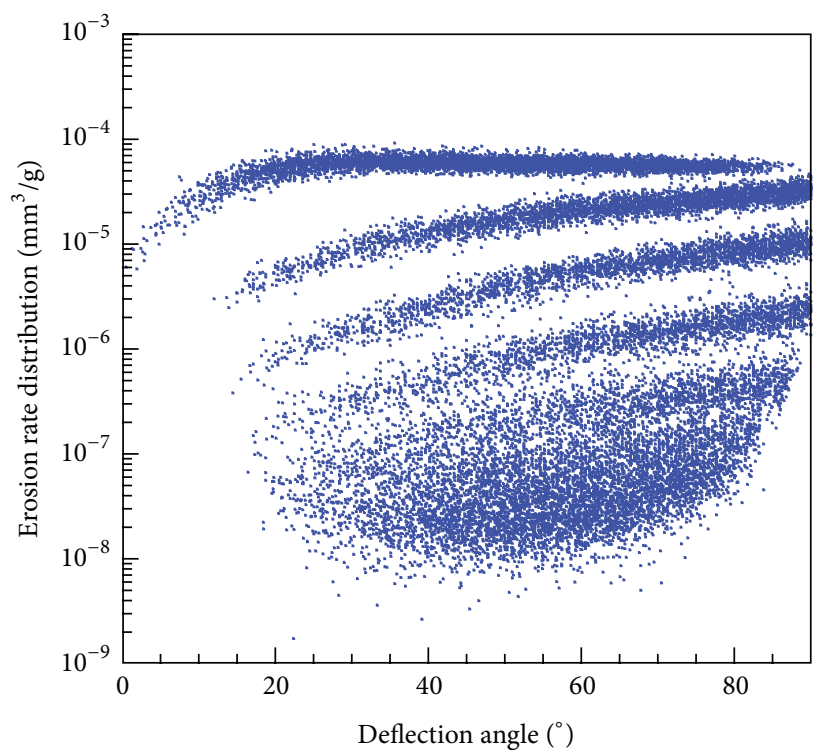

(a)

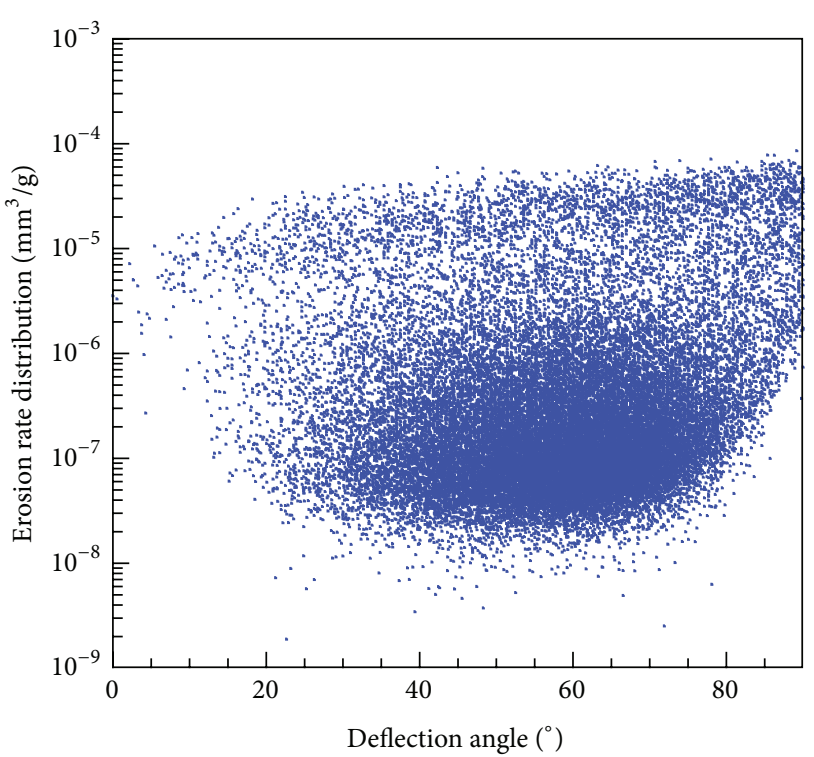

(b)

FiguRE 5: Erosion rate $E_{v}$ distribution per impact particle against the deflection angle of curved duct at the inlet velocity $5.3 \mathrm{~m} / \mathrm{s}:$ (a) particle diameter $60 \mu \mathrm{m}$ and (b) particle diameter $30 \mu \mathrm{m}$.

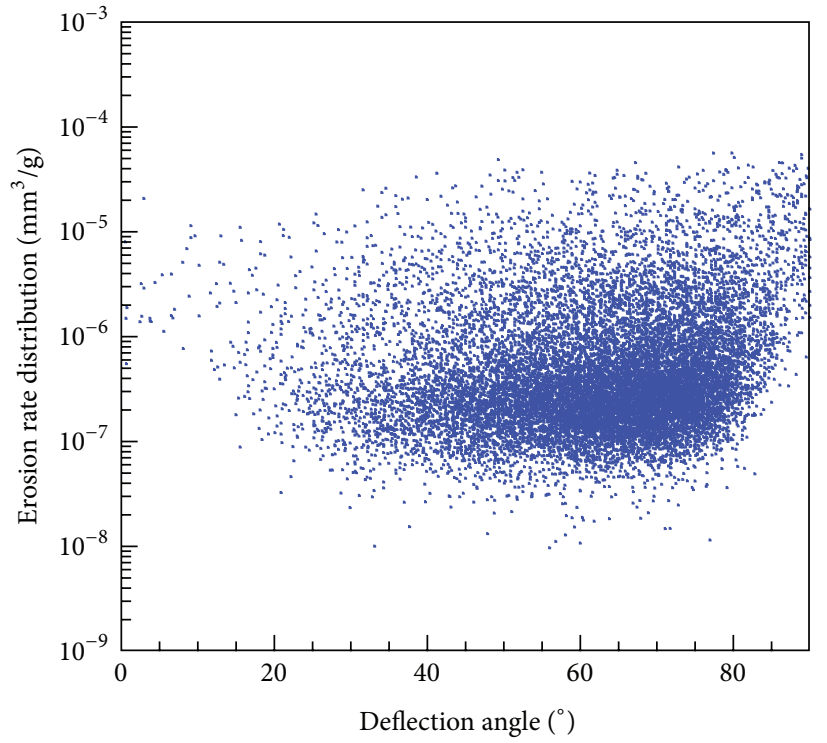

(a)

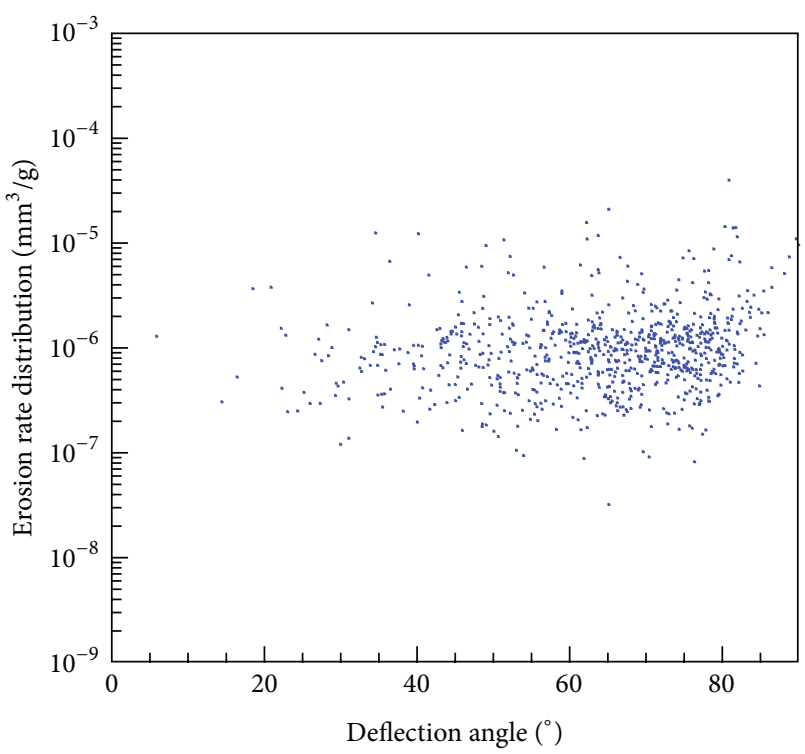

(b)

FIGURE 6: Erosion rate $E_{v}$ distribution per impact particle against the deflection angle of curved duct at the inlet velocity $5.3 \mathrm{~m} / \mathrm{s}$ : (a) particle diameter $16 \mu \mathrm{m}$ and (b) particle diameter $7 \mu \mathrm{m}$.

stripes do not have end tails at $90^{\circ}$ deflection angle or bend outlet.

Figure 5 demonstrates the erosion rates for particles with diameters $d_{p}=60$ and $30 \mu \mathrm{m}$. From Figure 4 to Figure 5(a), the stripe boundaries are gradually obscure, and they begin to widen their widths. From Figure 5(a) to Figure 5(b), stripes have disappeared, and the general erosion rates decrease. In Figure 5(b), most of the erosions are from $E_{v}=1.0 \times 10^{-8}$ to $1.0 \times 10^{-6} \mathrm{~mm}^{3} / \mathrm{g}$. From
Figures 5 to 6, erosions decrease with impaction and deposition decreases because particle diameter decreases. From Figures 6 to 7, erosion amounts increase again as seen in Figures 2 and 3 due to diffusion-induced impactions of smaller particles. In Figure 7(b), most of the erosions are from $E_{v}=5.0 \times 10^{-7}$ to $1.0 \times 10^{-5} \mathrm{~mm}^{3} / \mathrm{g}$. These larger erosion values and amounts may be caused by larger incident angle impactions due to diffusion and turbulent eddy mixing. 


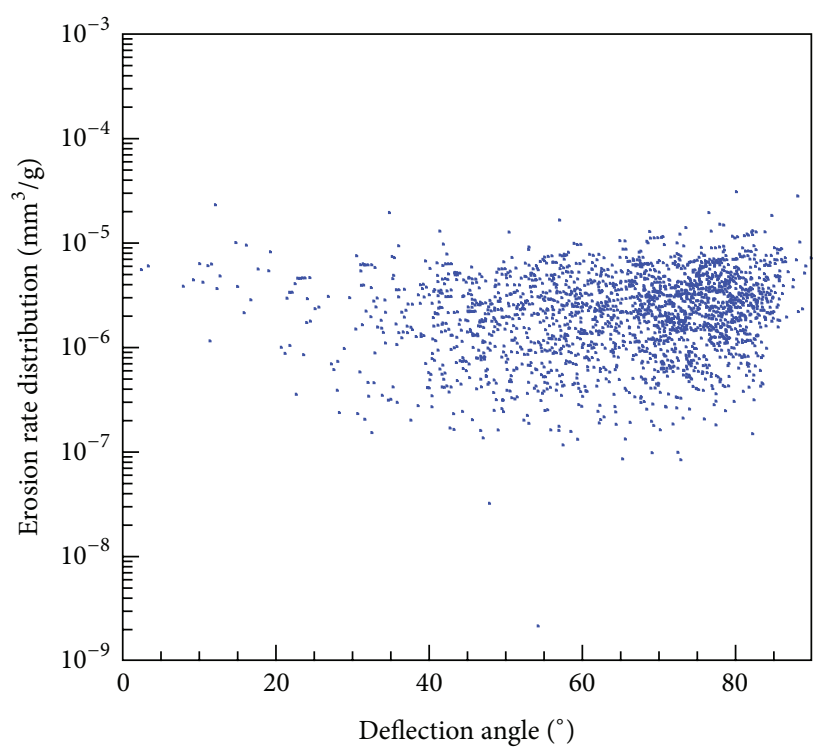

(a)

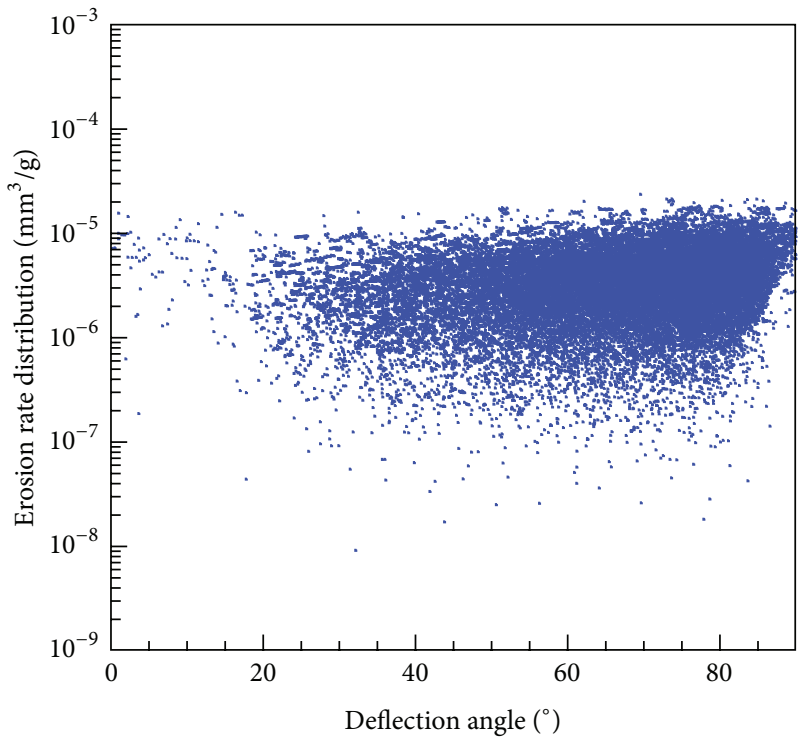

(b)

FiguRE 7: Erosion rate $E_{v}$ distribution per impact particle against the deflection angle of curved duct at the inlet velocity $5.3 \mathrm{~m} / \mathrm{s}$ : (a) particle diameter $3 \mu \mathrm{m}$ and (b) particle diameter $1 \mu \mathrm{m}$.

\section{Conclusions}

This paper presented a modeling and computational study on particle erosion in curved ducts. An algebraic particlewall impact model and a convenient erosion model were adopted in a typical 90-degree bend. The erosion rates per impacted particle and per injected particle were investigated against different particle diameter, inlet velocity, and Stokes number. The distribution of erosion rate was visualized and analyzed along bend deflection angles. The major findings are summarized as follows.

(1) Generally, the average erosion rates per impacted particles range from $4.2 \times 10^{-7}$ to $9.5 \times 10^{-3} \mathrm{~mm}^{3} / \mathrm{g}$ for the inlet velocity from $2.2 \mathrm{~m} / \mathrm{s}$ to $52 \mathrm{~m} / \mathrm{s}$. For each doubled inlet velocity, the increases of erosion rates per impact are 2-14 times. This phenomenon indicates a sharp enhancement of erosion rate due to inlet velocity increase.

(2) The erosion rate per impact changes with particle diameter with " $\sqrt{ }$ " shape in bend flows, which is similar to the particle deposition behavior in duct flows. When particle diameter $d_{p}$ is 7 or $16 \mu \mathrm{m}$, the erosion rate gets to the lowest value. Other smaller ones have higher erosion rates due to diffusion, turbulence, and vortex. Higher erosion rates for larger particles are caused by inertia and gravity.

(3) The erosion rate curves per injected particle are much like a 90-degree anticlockwise rotated "S" for three larger inlet velocities of 15,33 , and $52 \mathrm{~m} / \mathrm{s}$. The erosion rates range from $9.2 \times 10^{-5}$ to $2.0 \times 10^{-2} \mathrm{~mm}^{3} / \mathrm{g}$. The magnitudes of the values are 0.4-to-17.9 times larger than those per impact due to huge amounts of impacting, especially for those depositing particles. For the lower three inlet velocities, the erosion rate curves are much like wide open " $V$ " shapes. Their erosion rates range from $1.9 \times 10^{-7}$ to $3.7 \times 10^{-4} \mathrm{~mm}^{3} / \mathrm{g}$.

(4) It is obvious that the erosion rate is like a "fingerprint" with clear stripes for the three largest particles with diameters $d_{p}=60,100$, and $200 \mu \mathrm{m}$. The deflection angles with first impaction stripe are from $0^{\circ}$ to $60^{\circ}$, where the peak erosion rate appears at around $20^{\circ}$. After this impaction, other four clear impaction stripes are observed but with lower value due to the velocity reduction after each impaction. The lowest cloud-like areas are the lightest impactions close to particle depositions.

(5) For other smaller particles, the erosion rate distribution is much like an entire "cloud" along the bend deflection angle. These "clouds" become smaller and then bigger when the particle diameter decreases. The deflection angles of "clouds" are mainly from $20^{\circ}$ to $90^{\circ}$.

\section{Conflict of Interests}

The authors declare that there is no conflict of interests regarding the publication of this paper.

\section{Acknowledgment}

This work was financially supported by The Hong Kong Polytechnic University through research Grants 1-ZV6Z, APJ61, and A-PJ12. 


\section{References}

[1] M. R. Sippola and W. W. Nazaroff, "Particle deposition in ventilation ducts: connectors, bends and developing turbulent flow," Aerosol Science and Technology, vol. 39, no. 2, pp. 139-150, 2005.

[2] L. Jianzhong, S. Xing, and Y. Zhenjiang, "Effects of the aspect ratio on the sedimentation of a fiber in Newtonian fluids," Journal of Aerosol Science, vol. 34, no. 7, pp. 909-921, 2003.

[3] M. Yu, A. J. Koivisto, K. Hämeri, and M. Seipenbusch, "Size dependence of the ratio of aerosol coagulation to deposition rates for indoor aerosols," Aerosol Science and Technology, vol. 47, no. 4, pp. 427-434, 2012.

[4] T. Sarver, A. Al-Qaraghuli, and L. L. Kazmerski, "A comprehensive review of the impact of dust on the use of solar energy: history, investigations, results, literature, and mitigation approaches," Renewable and Sustainable Energy Reviews, vol. 22, pp. 698-733, 2013.

[5] K. Sun and L. Lu, "Particle flow behavior of distribution and deposition throughout $90^{\circ}$ bends: analysis of influencing factors," Journal of Aerosol Science, vol. 65, pp. 26-41, 2013.

[6] J. Z. Lin, P. F. Lin, and H. J. Chen, "Research on the transport and deposition of nanoparticles in a rotating curved pipe," Physics of Fluids, vol. 21, no. 12, Article ID 122001, 11 pages, 2009.

[7] S. M. El-Behery, M. H. Hamed, K. A. Ibrahim, and M. A. El-Kadi, "CFD evaluation of solid particles erosion in curved ducts," Journal of Fluids Engineering, vol. 132, no. 7, Article ID 071303, 10 pages, 2010.

[8] K. Sun, L. Lu, and H. Jiang, "A numerical study of bend-induced particle deposition in and behind duct bends," Building and Environment, vol. 52, pp. 77-87, 2012.

[9] X. H. Chen, B. S. McLaury, and S. A. Shirazi, "A comprehensive procedure to estimate erosion in elbows for gas/liquid/sand multiphase flow," Journal of Energy Resources Technology, vol. 128, no. 1, pp. 70-78, 2006.

[10] Y. L. Zhang, B. S. McLaury, and S. A. Shirazi, "Improvements of particle near-wall velocity and erosion predictions using a commercial CFD code," Journal of Fluids Engineering, vol. 131, no. 3, Article ID 031303, 9 pages, 2009.

[11] R. Macchini, M. S. A. Bradley, and T. Deng, "Influence of particle size, density, particle concentration on bend erosive wear in pneumatic conveyors," Wear, vol. 303 , no. 1-2, pp. 2129, 2013.

[12] ANSYS Inc., ANSYS FLUENT 12.0 Users Guide, ANSYS Inc., Lebanon, NH, USA, 2010.

[13] J. Z. Lin, J. Li, and W. F. Zhang, "The forces exerted on a cylindrical particle in the elongational-shear flows," International Journal of Nonlinear Sciences and Numerical Simulation, vol. 5, no. 1, pp. 9-16, 2004.

[14] B. Zhao, Y. Zhang, X. T. Li, X. D. Yang, and D. T. Huang, "Comparison of indoor aerosol particle concentration and deposition in different ventilated rooms by numerical method," Building and Environment, vol. 39, no. 1, pp. 1-8, 2004.

[15] D. I. Graham and P. W. James, "Turbulent dispersion of particles using eddy interaction models," International Journal of Multiphase Flow, vol. 22, no. 1, pp. 157-175, 1996.

[16] R. M. Brach and P. F. Dunn, "Models of rebound and capture for oblique microparticle impacts," Aerosol Science and Technology, vol. 29, no. 5, pp. 379-388, 1998.

[17] M. Menguturk and E. F. Sverdrup, "Calculated tolerance of a large electric utility gas turbine to erosion damage by coal gas ash particles," ASTM Special Technical Publications 664, ASTM, Philadelphia, Pa, USA, 1979.

[18] X. Q. Song, J. Z. Lin, J. F. Zhao, and T. Y. Shen, "Research on reducing erosion by adding ribs on the wall in particulate twophase flows," Wear, vol. 193, no. 1, pp. 1-7, 1996.

[19] K. Sun, L. Lu, H. Jiang, and H. Jin, "Experimental study of solid particle deposition in $90^{\circ}$ ventilated bends of rectangular cross section with turbulent flow," Aerosol Science and Technology, vol. 47, no. 2, pp. 115-124, 2013.

[20] M. R. Sippola, Particle deposition in ventilation ducts [Ph.D. thesis], University of California, Berkeley, Berkeley, Calif, USA, 2002.

[21] A. C. K. Lai and W. W. Nazaroff, "Modeling indoor particle deposition from turbulent flow onto smooth surfaces," Journal of Aerosol Science, vol. 31, no. 4, pp. 463-476, 2000.

[22] F. M. White, Fluid Mechanics, McGraw-Hill, New York, NY, USA, 2nd edition, 1986.

[23] M. Breuer, H. T. Baytekin, and E. A. Matida, "Prediction of aerosol deposition in $90^{\circ}$ bends using LES and an efficient Lagrangian tracking method," Journal of Aerosol Science, vol. 37, no. 11, pp. 1407-1428, 2006. 


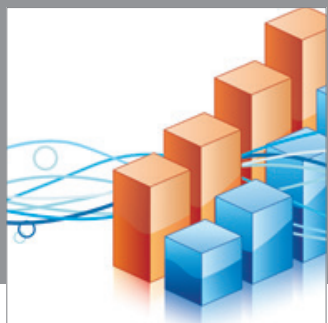

Advances in

Operations Research

mansans

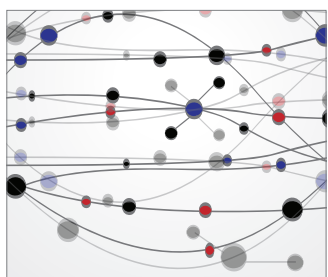

The Scientific World Journal
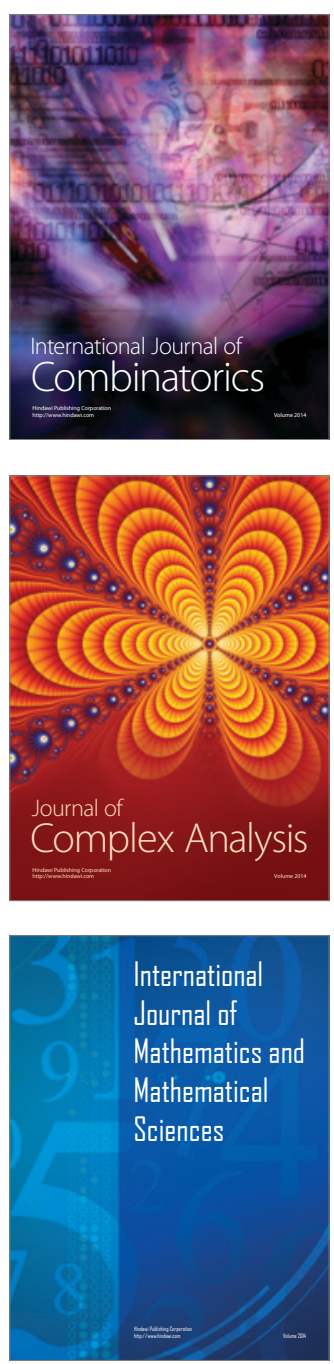
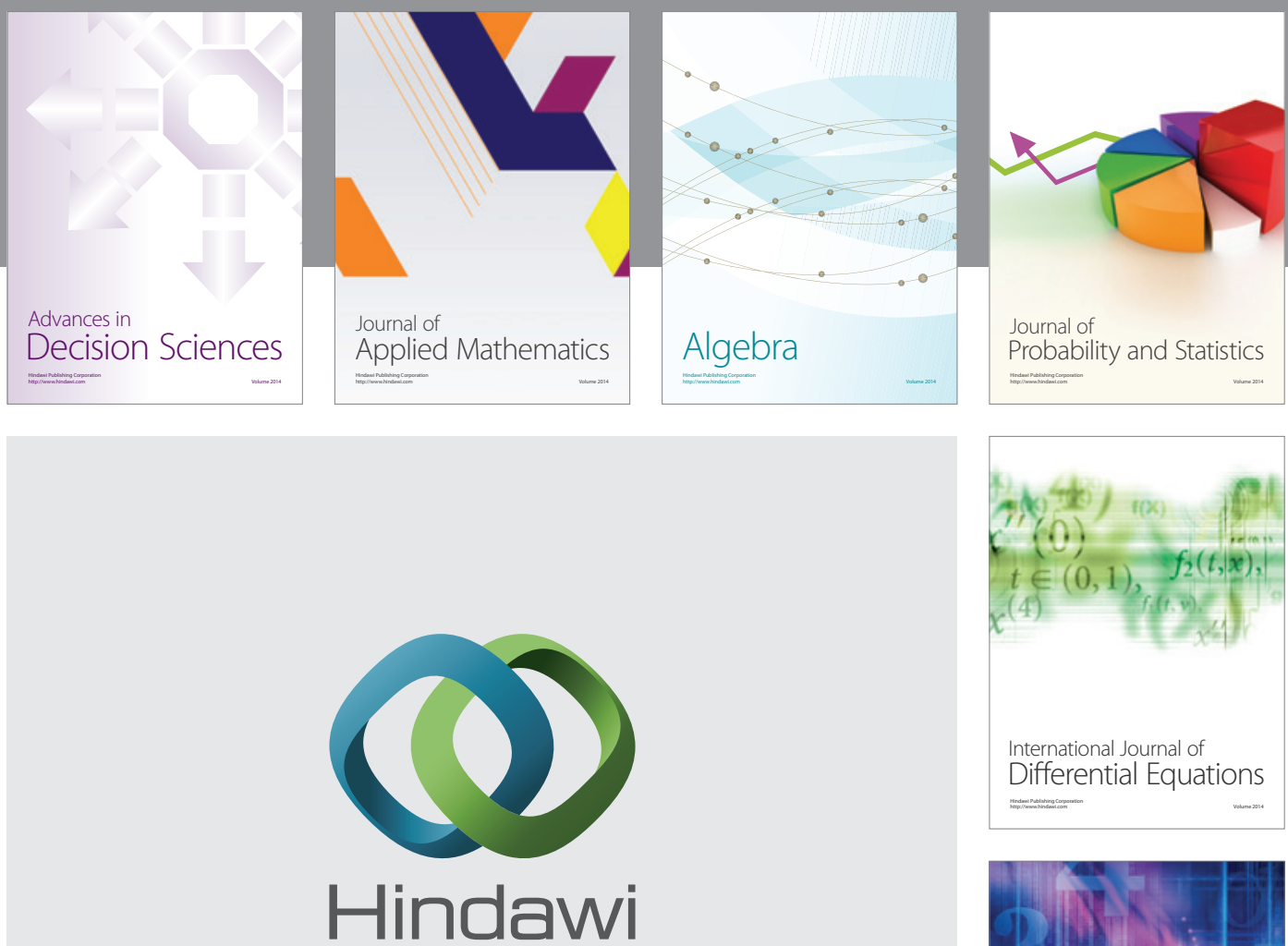

Submit your manuscripts at http://www.hindawi.com
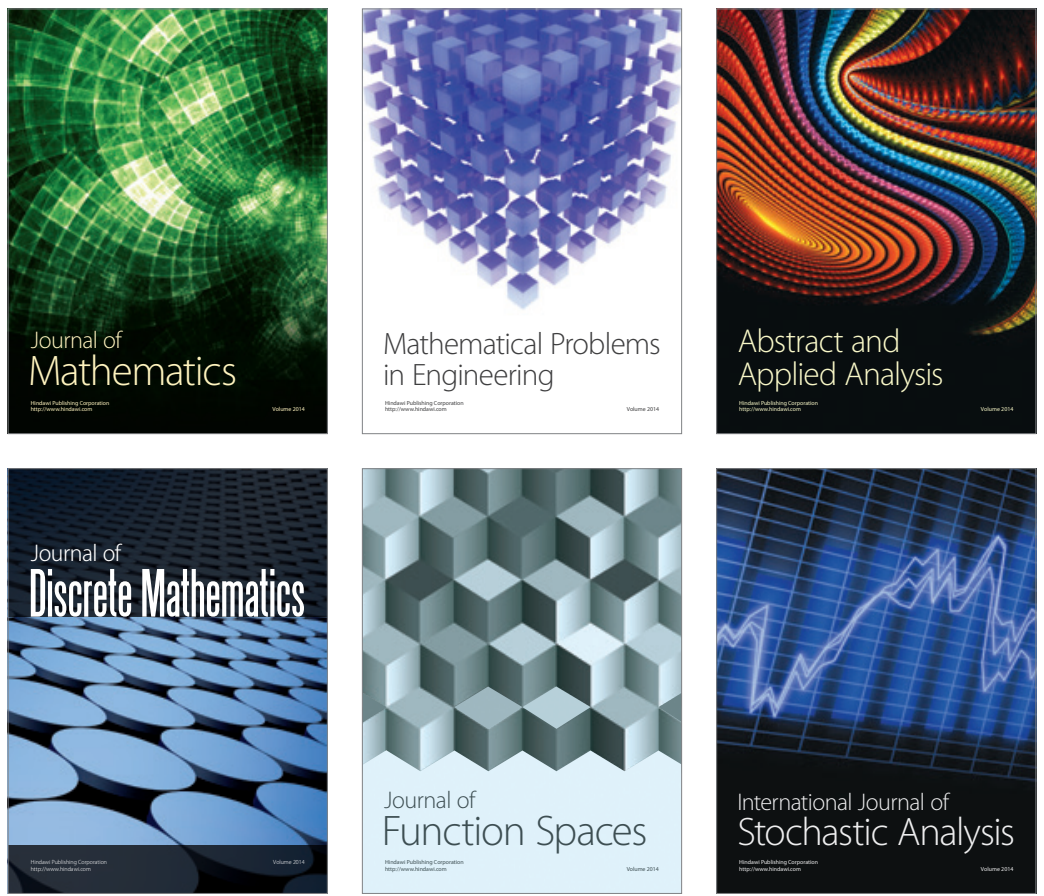

Journal of

Function Spaces

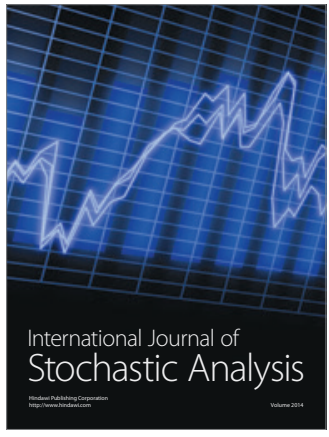

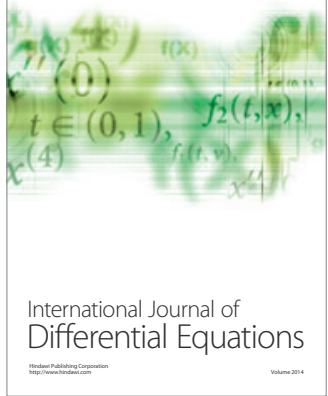
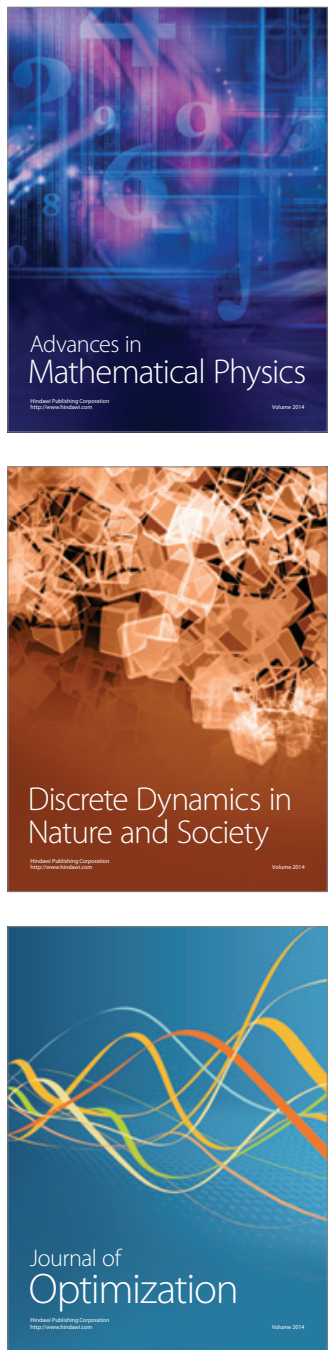